XLVIth Zakopane School of Physics, International Symposium Breaking Frontiers, Zakopane, Poland, May 16-21, 2011

\title{
EUV-Induced Nanostructuring of Solids
}

\author{
A. Bartnik*, H. Fiedorowicz, R. Jarocki, J. Kostecki, A. Szczurek, M. Szczurek
}

\author{
AND P. WACHULAK
}

Institute of Optoelectronics, Military University of Technology, S. Kaliskiego 2, 00-908 Warsaw, Poland

\begin{abstract}
In this work results of investigations concerning nanostructuring of polymers and some other solids using a laser-plasma extreme ultraviolet source are presented. The plasma radiation was produced using a gas puff target and focused with a gold-plated grazing incidence ellipsoidal collector. Decomposition process of polymers was investigated using a quadrupole mass spectrometer. Different kinds of micro- and nanostructures created in near-surface layers of the materials were investigated using scanning electron microscope. Forms of the structures depend on a particular material and the extreme ultraviolet exposure. In case of some polymers even a single shot is sufficient for creation of the visible changes in surface morphology. In case of inorganic solids visible changes require usually the exposure with tens or hundreds of extreme ultraviolet pulses.
\end{abstract}

PACS: 52.38.Ph, 82.50.Kx, 81.40.Wx

\section{Introduction}

Plasma or UV radiation treatment of polymers can result in formation of different kinds of micro- or nanostructures in the near surface layer. Many works were devoted to such modification using excimer lamps or excimer lasers. Photons emitted from these sources can excite electrons from the valence band of any organic material. The excited state can relax through a radiative or nonradiative process. In case of polymers one of the possible radiationless channels is bond breaking of a polymer chain, other lead to increase of the polymer temperature. As a result polymer chains become shorter and their chemical structure and composition can change.

Some volatile fractions can be released from the irradiated layer. The surface morphology can change due to preferential dry etching of amorphous polymer or melting of the near surface layer combined with a transient pressure of the ablation plume. Forms and characteristic sizes of surface structures depend both on radiation and material properties. UV absorption in a particular polymer is strongly dependent on the radiation wavelength.

It was shown in [1] that absorption depth for a $\mathrm{KrF}$ excimer laser $(284 \mathrm{~nm})$ varies from 10 to $500 \mu \mathrm{m}$. If the wavelength is properly adjusted to a polymer absorption properties, the penetration depth can be less than $1 \mu \mathrm{m}$ $[2,3]$. A very important parameter is also the radiation fluence. If the fluence is high, well above the ablation threshold, the surface modification is less important, because most of the material degraded by the UV radiation is removed from the surface [2]. In case of relatively low fluence close to the ablation threshold self-organized micro- or nanostructuring on the irradiated polymer surface can occur.

In many papers formation of different kinds of microstructures was reported. These are usually conical

\footnotetext{
* corresponding author; e-mail: abartnik@wat.edu.pl
}

$[4,5]$ wall- or nap-type $[6,7]$ structures. Irradiation of polystyrene (PS) with $\mathrm{KrF}$ laser close to the ablation threshold resulted in formation of surface ripple structures with sub- $\mu$ m periodicity $[8,9]$. Such modification of surface morphology accompanied by some chemical changes is very important in a wide spectrum of biotechnologies. A very important issue here is biocompatibility of polymers. It is connected with cell adhesion and proliferation on the polymer surface. It strongly depends on chemical and physical properties of the surface. Various surface structures in micro- and nanoscale were applied to study correlation between surface topography and cell adhesion [9-11].

Surface modification can be also obtained using extreme ultraviolet (EUV) instead of UV irradiation. The EUV photons can excite or release any electron from the valence band. Such photons are thus strongly absorbed in any polymer. The smallest absorption depth for polyethylene terephthalate (PET) or polymers having similar composition is approximately $20 \mathrm{~nm}$ for photons with energy of $20 \mathrm{eV}$ [12].

The absorption depth increases with photon energy up to about $200 \mathrm{~nm}$ for $100 \mathrm{eV}$ but is still much smaller comparing to absorption depth of UV radiation. In opposite to UV radiation the absorption depth of EUV radiation in polymers hardly depends on a polymer structure and chemical composition. Thus the polymer material in the bulk is not affected by the EUV radiation. In this paper we present our recent results concerning surface modifications of different kinds of polymers and inorganic solids.

\section{Laser-plasma EUV source}

In the experiments, a $10 \mathrm{~Hz}$ laser plasma EUV source based on a double stream gas puff target was used. The target was irradiated using 4 ns Nd:YAG laser pulses with energy $0.8 \mathrm{~J}$. The double stream gas puff target was created by pulsed injection of xenon or krypton into a hollow stream of helium with the use of an electromagnetic valve system equipped with a double nozzle setup. 
The outer stream of gas (the buffer gas) confines the inner gas stream and prevents its spherical expansion outside the nozzle. This way the inner gas has a form of an elongated stream with relatively high density and a sharp density gradient at the boundary with the outer gas. It makes possible to focus a laser beam in the high density region of gas far away from the nozzle outlet and achieve an efficient EUV production without degradation of the nozzle.

The EUV radiation was focused using a gold-plated grazing incidence ellipsoidal collector, manufactured in Reflex s.r.o. (at present: Rigaku Innovative Technologies Europe s.r.o.), Czech Republic. The collector allowed for effective focusing of radiation emitted from $\mathrm{Kr}$ or Xe plasmas in the wavelength range $\lambda=9 \div 70 \mathrm{~nm}$. The most intense emission was in the relatively narrow spectral region centred at $\lambda=10 \pm 1 \mathrm{~nm}$. The spectral intensity at longer wavelength range was much smaller, however, the spectrally integrated intensities in both ranges were comparable. The EUV fluence in the focal plane of the collector exceeded $60 \mathrm{~mJ} / \mathrm{cm}^{2}$ at the centre of the focal spot. FWHM diameter of the intensity distribution in the focal spot was $1.4 \mathrm{~mm}$. Detailed description of the source and parameters of the focused EUV radiation can be found elsewhere [13]. The main disadvantage is the attenuation of EUV in a residual gas filling the vacuum chamber during operation with high repetition. To minimize the absorption an EUV collector was mounted in an additional chamber and a differential pumping arrangement was applied.

\section{Experimental results}

\subsection{Decomposition products}

Irradiation of chemical compounds with EUV radiation results in bond breaking and in consequence decomposition of molecules. In case of polymers it can lead to shortening of the molecular chains or formation of volatile products that can be trapped in the near surface layer or can be ejected from the surface. In both cases morphology of the irradiated surface can change leading to formation of self-organized micro- or nanostructures. The small molecules or radicals released this way to the vacuum chamber can be investigated using mass spectrometry.

In this work quadrupole mass spectrometer (QMS) spectra were acquired for different kinds of polymers irradiated with different EUV intensities. The EUV intensity was adjusted by changing the distance of the irradiated sample from the focal plane of the EUV collector. The longer distance downstream the focal plane the larger surface was irradiated with lower fluence preserving the total amount of EUV photons reaching the polymer surface. For a particular polymer the QMS measurements indicate significant decrease of emission of the decomposition products with decrease of EUV fluence. Some differences in spectral distributions were also revealed.

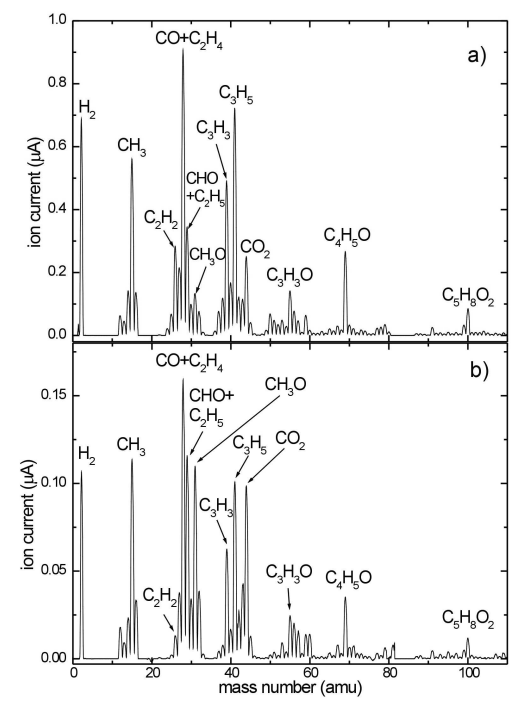

Fig. 1. QMS spectra of decomposition products ejected from PMMA surface during EUV irradiation with different fluences in a single pulse: (a) $\geq 40$ $\mathrm{mJ} / \mathrm{cm}^{2},(\mathrm{~b}) \leq 10 \mathrm{~mJ} / \mathrm{cm}^{2}$.

As an example QMS spectra obtained for polymethyl methacrylate (PMMA) were shown in Fig. 1. The spectra acquired in the mass range 1-110 amu consist of multiple peaks corresponding to decomposition products with different molecular masses. Repeating structural unit of PMMA polymer is relatively simple containing only three kinds of atoms, namely $\mathrm{H}, \mathrm{C}$ and $\mathrm{O}$. The most characteristic peaks are thus easy to identify, however, some of them can be attributed to more than one molecular species.

For example, the most intense peak corresponding to the mass number 28 can be associated with emission of both $\mathrm{CO}$ and $\mathrm{C}_{2} \mathrm{H}_{4}$ molecules. The other peaks can be attributed to different kinds of fragments of the PMMA chain. One of them corresponding to a relatively high molecular mass equal to $100 \mathrm{amu}$ can be attributed to the complete repeating unit of the PMMA chain $\mathrm{C}_{5} \mathrm{H}_{8} \mathrm{O}_{2}$. Intensity of the peak $I_{100}$ is not very high in relation to the most intense one $I_{28}\left(I_{100} / I_{28} \approx 0.1\right)$ but taking into account masses corresponding to both peaks the ratio $100 I_{100} / 28 I_{28}$ accounts for about 0.35 .

Three other pronounced peaks having also relatively high molecular masses are associated with emission of $\mathrm{C}_{3} \mathrm{H}_{5}, \mathrm{C}_{3} \mathrm{H}_{3} \mathrm{O}$ and $\mathrm{C}_{4} \mathrm{H}_{5} \mathrm{O}$ molecules being parts of the repeating unit. Emission of these molecules as well as the entire repeating unit requires breaking of the polymer backbone. Their emission is thus associated mainly with shortening of the polymer chains leading to efficient material ablation.

There are, however, considerable differences between the QMS spectra corresponding to irradiation of PMMA with high and low fluences. Examples of the spectra for PMMA samples irradiated at the distances 3 and $15 \mathrm{~mm}$ downstream the focal plane were shown in Fig. 1a and b, 
respectively. EUV fluence in the first case significantly exceeded the ablation threshold while in the second case was close to this threshold.

It should be noticed that relative intensities of most of the peaks in the first spectrum are approximately 6 times higher than in the second one. High emission of decomposition products is associated with intense material ablation as could be expected for high EUV fluence. Low emission is rather associated with material desorption accompanied by surface modification.

There are, however, three intense peaks in the second spectrum corresponding to emission of $\mathrm{CHO}, \mathrm{CH}_{3} \mathrm{O}$, and $\mathrm{CO}_{2}$ species with much higher relative intensities in respect of the first spectrum. These species are fragments of the pendant groups of the PMMA chain. Their detachment does not require breaking of the polymer backbone. It suggests that in case of low fluence such decomposition scheme is preferential and leads to significant modification of chemical structure of the polymer and in consequence changes of the ablation threshold in the near surface layer. Usually these changes are non-uniform, hence, further irradiation results in formation of self-organized micro- and nanostructures.

\subsection{Surface morphology}

Emission of different decomposition products from irradiated PMMA proves fragmentation of the polymer chains or detachment of some pendant groups. Differences in the QMS spectra for low and high irradiation fluence are associated with differences of chemical structures in the near surface layers after irradiation. Some decomposition products remaining in the near surface layers are soluble in organic solvents, hence, rinsing of the samples irradiated with different conditions can result in different surface morphologies.

Examples of such morphologies obtained for PMMA irradiated with high and low fluence and rinsed in typical PMMA developer (isobutylmethyl ketone +2 -propanol, 1:3 mixture) are shown in Fig. 2a,b. The structure shown in Fig. 2a was obtained in the PMMA sample irradiated with 5 EUV pulses of high fluence, while the structure in Fig. 2b was obtained with low fluence but 100 EUV pulses. In both cases nanocavities in the near surface layer are clearly visible. Their form and number is a little bit different, however, the differences are not essential.

Dramatic differences can be seen in case of PET irradiated with high and low fluence. In the first case (Fig. 2c) irradiation with 2 pulses followed by acetone rinsing results in a granular surface structure with the characteristic size less than $100 \mathrm{~nm}$. Rinsing of the PET sample irradiated with 25 EUV pulses of the fluence close to ablation threshold results in a worm-like structure shown in Fig. 2d. Characteristic size of the structure is approximately $200 \mathrm{~nm}$. The size depends to some extent on the exact EUV fluence. The surface structure of both materials before irradiation and rinsing was smooth in this scale. Also irradiation without further rinsing performed in the same conditions as mentioned above does not give
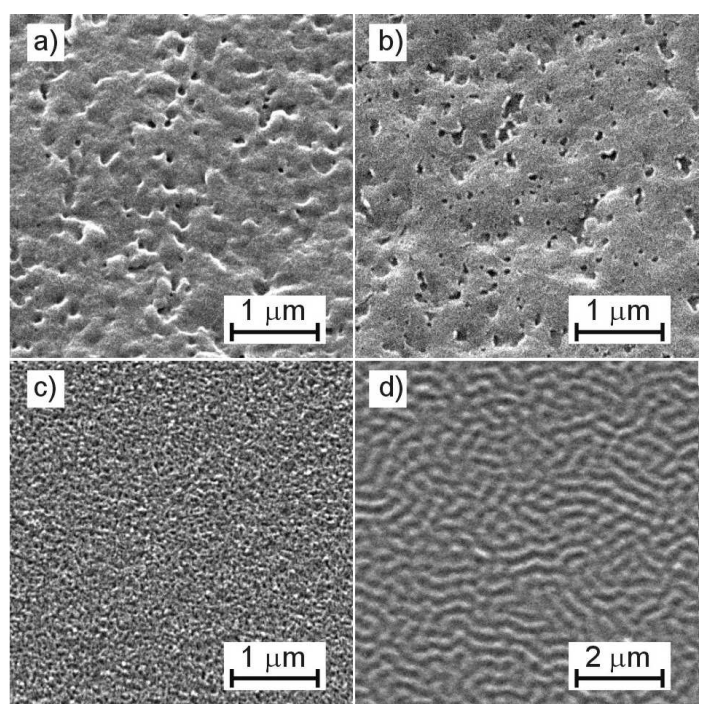

Fig. 2. Nanostructures formed in polymers irradiated and rinsed in different conditions: (a) PMMA, 5 EUV pulses with maximum fluence, rinsed in typical PMMA developer, (b) PMMA, 100 EUV pulses with low fluence, rinsed in PMMA developer, (c) PET, 2 EUV pulses with maximum fluence, rinsed in acetone, (d) PET, $25 \mathrm{EUV}$ pulses with low fluence, rinsed in acetone.

such structures. Formation of the nanostructures is thus a combined effect of EUV irradiation and removal of the soluble fractions.

On the other hand, formation of nanostructures on a polymer surface can be obtained without further rinsing, however, it requires approximately 1 min EUV exposure with $10 \mathrm{~Hz}$ with intensity close to the ablation threshold.

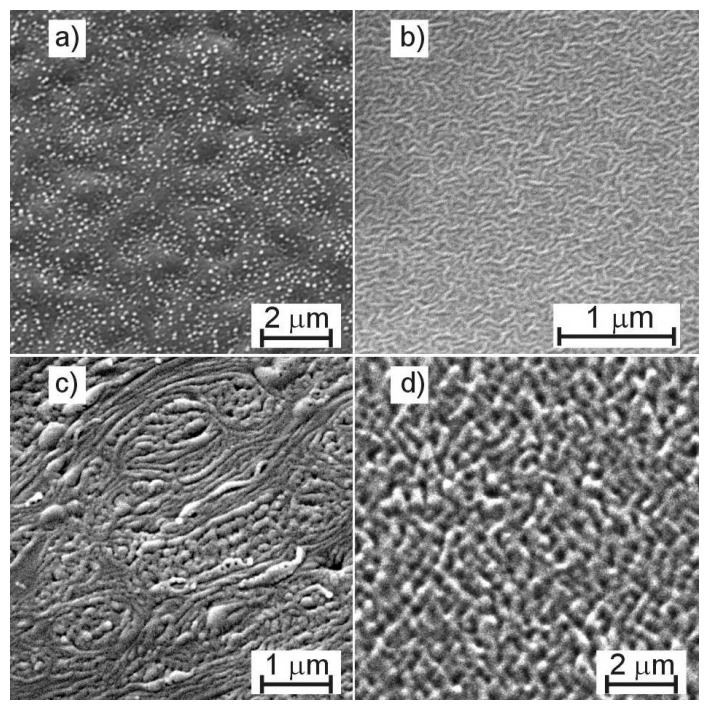

Fig. 3. Nanostructures formed in different polymers irradiated with low (a)-(c) and high intensity (d) with different number of EUV pulses: (a) PMMA - 600, (b) FEP - 600, (c) PET - 1200, (d) PEN - 5 pulses. 
Examples of the structures obtained this way are shown in Fig. 3a-c. In the first case (Fig. 3a) surface of a PMMA sample irradiated with 600 EUV pulses is covered with large number of nodules having the size of the order of $100 \div 200 \mathrm{~nm}$. Their origin is probably associated with production of small polymer fragments trapped locally in the near surface layer. The nodules could be created by volatilization just underneath the surface causing thereby surface swelling on the nm scale.

Quite different structures were obtained in fluorinated ethylene propylene (FEP) and PET (Fig. 3b, c). The FEP sample after 1 min irradiation with EUV intensity near the ablation threshold is covered with the worm-like structures similar to the nanostructures shown in Fig. 2d. Their characteristic lateral size, however, is much smaller, approximately $50 \mathrm{~nm}$. Concerning PET, 2 min irradiation of this polymer with intensity close to the ablation threshold results in much more complicated pattern with characteristic size of the structures $100 \div 200 \mathrm{~nm}$

Additionally, the structures are partially oriented in opposite to PMMA and FEP. This orientation is clearly visible in case of irradiation with a high fluence. In this case a quasi-periodic microstructure is being formed [14]. This structure is attributed to relaxation of frozen stress fields originating from the production process and is probably also responsible for partial orientation of the nanostructures shown in Fig. 3c.

An origin of the nanostructures formed in FEP and PET polymers is unclear. It can be associated with preferential dry etching of amorphous polymer combined with chemical changes in the near surface layer.

In case of some polymers like polystyrene (PS), polyvinylidene chloride (PVDC), or polyethylene naphthalate (PEN) irradiation even with a single pulse of high fluence without any further treatment can result in creation of nanostructures. An example of such a structure obtained in PEN is presented in Fig. 3d. In this case irradiation with 5 EUV pulses resulted in creation of a pronounced structure with a characteristic size of approximately $400 \mathrm{~nm}$. This structure is rather not oriented, however, further irradiation results in larger quasi-periodic well oriented structure. The nanostructure is thus an initial stage of a wall-like microstructure formation well described in $[7,15]$.

One of the most interesting polymers is polytetrafluoroethylene (PTFE). Many works were devoted to micromachining of this polymer using synchrotron X-rays $[16,17]$ because absorption of this material from infrared (IR) to ultraviolet (UV) is very weak. On the other hand, absorption of EUV radiation in PTFE is very strong. The absorption depth is less than $100 \mathrm{~nm}$, hence, micromachining of this material using EUV is possible and was demonstrated in $[18,19]$.

In this work PTFE was irradiated with the fluence close to the ablation threshold. After approximately 1 min irradiation the surface morphology becomes modified and cavities with the submicro sizes are formed

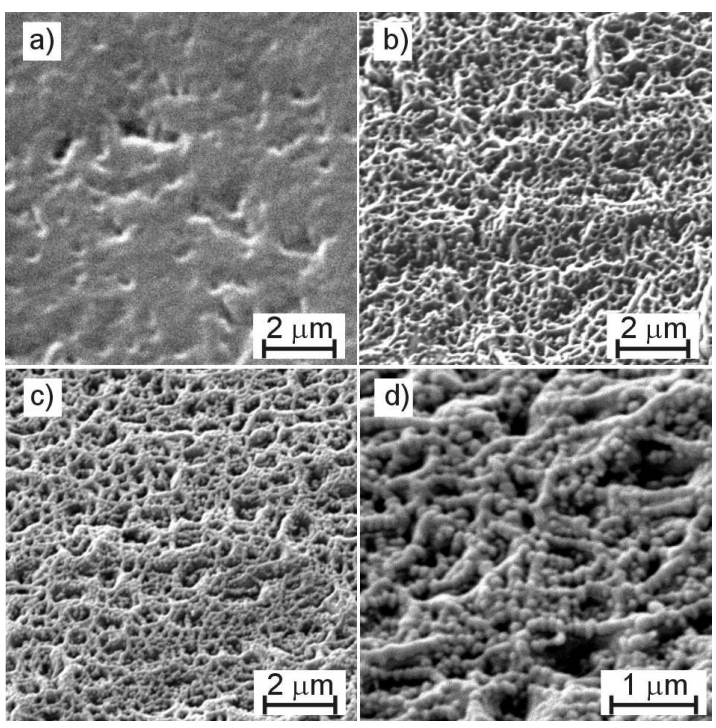

Fig. 4. Nanostructures formed in PTFE irradiated with low intensity and different EUV exposure times: (a) 1 min, (b) 2 min, (c,d) 3 min.

(Fig. 4a). 2 min irradiation results in formation of very complicated fibrous structure shown in Fig. 4b. This structure evolves during further irradiation leading to beads-on-a-string nanostructures and with characteristic size of beads $50 \div 100 \mathrm{~nm}$. The structure was shown in Fig. 4c,d.

Fluence of the EUV radiation employed for ablation of polymers is usually insufficient for ablation of inorganic materials because of much higher ablation threshold. In case of some inorganic materials, however, strong modification of the near surface layer is possible.

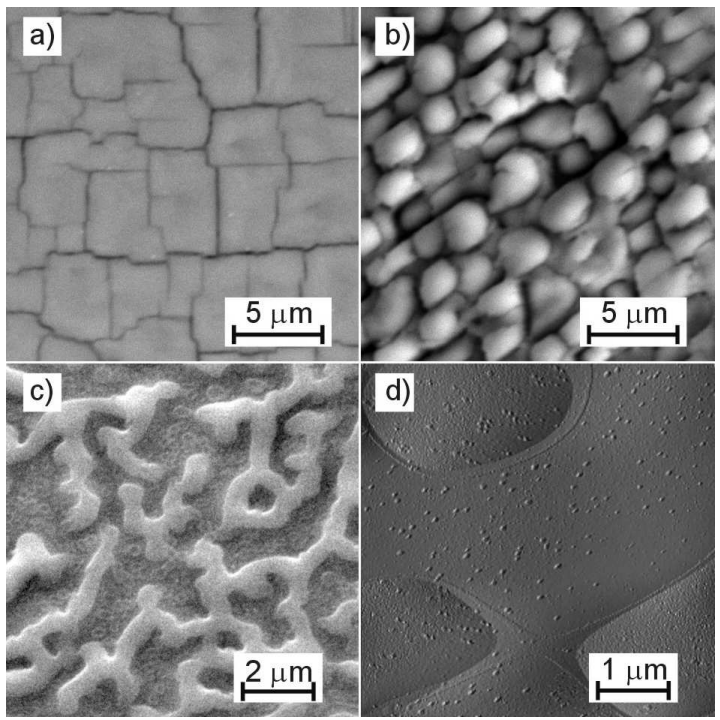

Fig. 5. Micro- and nanostructures formed in different inorganic monocrystals: (a) $\mathrm{NaCl} 1 \mathrm{~min}$, (b) $\mathrm{CaF}_{2}$ 1 min, (c) GaAs 10 s, (d) Ge 2 min. 
In Fig. 5 examples of micro- and nanostructures obtained in four different monocrystals are presented. The images shown in Fig. 5a, b are surfaces of $\mathrm{NaCl}$ and $\mathrm{CaF}_{2}$ monocrystals respectively after $1 \mathrm{~min}$ irradiation with $10 \mathrm{~Hz}$ repetition rate. In both cases thin surface layers were divided into microcrystals. The smallest crystal size shown here was about $2 \mu \mathrm{m}$ for $\mathrm{NaCl}$ and $1 \mu \mathrm{m}$ for $\mathrm{CaF}_{2}$. The mechanism governing creation of these structures is not clear.

The microcrystalline structure is created probably as a result of fast melting and recrystallization. It may also arise due to stresses connected with temperature gradient.

In Fig. 5c microstructure formed in GaAs monocrystal irradiated with 100 of EUV pulses is presented. Form of the structure suggests that their origin is associated with melting of the near surface layer. The other possibility is chemical decomposition of the material and melting of gallium (melting point $30^{\circ} \mathrm{C}$ ).

In the last image (Fig. 3d) surface of Ge monocrystal after 2 min irradiation with $10 \mathrm{~Hz}$ is shown. The resulting microstructure has a very small amplitude of the order of $20 \mathrm{~nm}$. Its origin is probably also associated with melting of the near surface layer with the thickness corresponding to attenuation length of EUV radiation in Ge.

\section{Conclusion}

In this paper we present the results of experiments concerning surface modification of organic polymers and inorganic monocrystals irradiated with EUV pulses. Decomposition of polymers was investigated using QMS and results obtained for PMMA are presented.

Different kinds of nanostructures were obtained in polymers after irradiation with small number of EUV pulses followed by rinsing in organic solvents. Irradiation with large number of EUV pulses without any further treatment also resulted in formation of different kinds of nanostructures. Such structures have not been reported in case of UV irradiation. Micro- and nanostructures were also obtained for different inorganic monocrystals. Surface modification of these materials required relatively high EUV fluence, close to the maximum value.

\section{Acknowledgments}

This research was supported by the Polish Ministry of Science and Higher Education under the projects No. N N202 174939, No. 816/N-COST/2010 and the European
Commission's Seventh Framework Program (Laserlab Europe, and COST - European Cooperation in Science and Technology - Action MP0601).

\section{References}

[1] N.S. Murthy, R.D. Prabhu, J.J. Martin, L. Zhou, R.L. Headrick, J. Appl. Phys. 100, 023538 (2006).

[2] T. Lippert, Adv. Polym. Sci. 168, 51 (2004).

[3] S. Lazare, R. Srinivasan, J. Phys. Chem. 90, 2124 (1986).

[4] B. Hopp, Zs. Bor, E. Homolya, E. Mihalik, Appl. Surf. Sci. 109/110, 232 (1997).

[5] V. Oliveira, R. Vilar, Appl. Phys. A 92, 957 (2008).

[6] N. Bityurin, E. Arenholz, N. Arnold, D. Bäuerle, Phys. Rev. E 75, 041603 (2007).

[7] E. Arenholz, V. Svorcik, T. Kefer, J. Heitz, D. Bauerle, Appl. Phys. A 53, 330 (1991).

[8] T. Peterbauer, S. Yakunin, J. Siegel, S. Hering, M. Fahrner, C. Romanin, J. Heitz, J. Nanomater. 2011, 413079 (2010).

[9] E. Rebollar, I. Frischauf, M. Olbrich, T. Peterbauer, S. Hering, J. Preiner, P. Hinterdorfer, C. Romanin, J. Heitz, Biomaterials 29, 1796 (2008).

[10] Y. Wan, Y. Wang, Z. Liu, X. Qu, B. Han, J. Bei, S. Wang, Biomaterials 26, 4453 (2005).

[11] E.K.F. Yim, R.M. Reano, S.W. Pang, A.F. Yee, C.S. Chen, K.W. Leong, Biomaterials 26, 5405 (2005).

[12] http://www-cxro.lbl.gov/.

[13] A. Bartnik, H. Fiedorowicz, R. Jarocki, J. Kostecki, A. Szczurek, M. Szczurek, Appl. Phys. B 96, 727 (2009).

[14] A. Bartnik, H. Fiedorowicz, R. Jarocki, J. Kostecki, M. Szczurek, A. Biliński, O. Chernyayeva, J.W. Sobczak, Appl. Phys. A 99, 831 (2010).

[15] J. Heitz, E. Arenholz, D. Bauerle, R. Sauerbrey, H.M. Phillips, Appl. Phys. A 59, 289 (1994).

[16] Y. Zhang, T. Katoh, M. Washio, H. Yamada, S. Hamada, Appl. Phys. Lett. 67, 872 (1995).

[17] T. Katoh, D. Yamaguchi, Y. Satoh, S. Ikeda, Y. Aoki, M. Washio, Y. Tabata, Appl. Surf. Sci. 186, 24 (2002).

[18] A. Bartnik, H. Fiedorowicz, R. Jarocki, L. Juha, J. Kostecki, R. Rakowski, M. Szczurek, Microelectron. Eng. 78-79, 452 (2005).

[19] A. Bartnik, H. Fiedorowicz, R. Jarocki, L. Juha, J. Kostecki, R. Rakowski, M. Szczurek, Appl. Phys. B 82, 529 (2006). 